\title{
GEOMETRIC WEAKLY ADMISSIBLE MESHES, DISCRETE LEAST SQUARES APPROXIMATIONS AND APPROXIMATE FEKETE POINTS
}

\author{
L. BOS, J.-P. CALVI, N. LEVENBERG, A. SOMMARIVA, AND M. VIANELLO
}

\begin{abstract}
Using the concept of Geometric Weakly Admissible Meshes (see $\S 2$ below) together with an algorithm based on the classical $\mathrm{QR}$ factorization of matrices, we compute efficient points for discrete multivariate least squares approximation and Lagrange interpolation.
\end{abstract}

\section{INTRODUCTION}

In a recent paper [8], Calvi and Levenberg have developed the theory of "admissible meshes" for uniform polynomial approximation over multidimensional compact sets. We begin by introducing some notation.

Suppose that $K \subset \mathbb{C}^{d}$ is compact. We will require that $K$ not be too small, that is, that it is polynomial determining; i.e., if a polynomial $p$ is zero on $K$, then it is identically zero. This will certainly be the case if $K$ contains some open real ball, as will be the case for all our examples.

We will let $\mathbb{P}_{n}^{d}$ denote the space of polynomials of degree at most $n$ in $d$ variables.

Then, a Weakly Admissible Mesh (WAM) for $K$ is a sequence of discrete subsets $A_{n} \subset K$ such that

$$
\|p\|_{K} \leq C\left(A_{n}\right)\|p\|_{A_{n}}, \quad \forall p \in \mathbb{P}_{n}^{d}
$$

where $\operatorname{card}\left(A_{n}\right)$ and the constants $C\left(A_{n}\right)$ have (at most) polynomial growth in $n$, i.e.,

$$
N:=\operatorname{dim}\left(\mathbb{P}_{n}^{d}\right)=\left(\begin{array}{c}
n+d \\
d
\end{array}\right) \leq \operatorname{card}\left(A_{n}\right)=\mathcal{O}\left(n^{\alpha}\right), \alpha>0
$$

and

$$
C\left(A_{n}\right)=\mathcal{O}\left(n^{\beta}\right), \quad \beta>0 .
$$

Throughout the paper, $\|p\|_{X}=\max _{\mathbf{x} \in X}|p(\mathbf{x})|$. When $\left\{C\left(A_{n}\right)\right\}$ is bounded, $C\left(A_{n}\right)$ $\leq C$, we speak of an Admissible Mesh (AM). It is easy to see that (W)AMs satisfy the following properties (cf. [8]):

Received by the editor February 10, 2009 and, in revised form, April 18, 2010.

2010 Mathematics Subject Classification. Primary 41A10, 41A63, 65D05, 65D15, 65 Y 20.

Key words and phrases. Admissible meshes, discrete least squares approximation, approximate Fekete points, multivariate polynomial interpolation.

The first author was supported in part by NSERC.

The last three authors were supported by the "ex-60\%" funds and by the project "Interpolation and Extrapolation: new algorithms and applications" (2009/10) of the University of Padova, and by the INdAM GNCS. 
- for affine mappings of $K$ the image of a WAM is also a WAM, with the same constant $C\left(A_{n}\right)$,

- any sequence of sets containing $A_{n}$, with polynomially growing cardinalities, is a WAM with the same constants $C\left(A_{n}\right)$,

- any sequence of unisolvent interpolation sets whose Lebesgue constant grows at most polynomially with $n$ is a WAM, $C\left(A_{n}\right)$ being the Lebesgue constant itself,

- a finite union of WAMs is a WAM for the corresponding union of compacts, $C\left(A_{n}\right)$ being the maximum of the corresponding constants,

- a finite cartesian product of WAMs is a WAM for the corresponding product of compacts, $C\left(A_{n}\right)$ being the product of the corresponding constants.

As shown in [8, such meshes are very useful for polynomial approximation in the max-norm on $K$. In fact, given the classical least-square polynomial approximant on $A_{n}$ to a function $f \in C(K)$, say $\mathcal{L}_{n} f \in \mathbb{P}_{n}^{d}$, we have that

$$
\left\|f-\mathcal{L}_{n} f\right\|_{K} \leq\left(1+C\left(A_{n}\right)\left(1+\sqrt{\operatorname{card}\left(A_{n}\right)}\right)\right) \min \left\{\|f-p\|_{K}, p \in \mathbb{P}_{n}^{d}\right\}
$$

see Theorem 1 of [8]. Moreover, Fekete points (points that maximize the Vandermonde determinant) extracted from a WAM have a Lebesgue constant with the bound

$$
\Lambda_{n} \leq N C\left(A_{n}\right),
$$

that is, $C\left(A_{n}\right)$ times the classical bound for the Lebesgue constant of true (continuum) Fekete points; see $\S 4.4$ of [8]. Recently, a new algorithm has been proposed for the computation of Approximate Fekete Points, using only standard tools of numerical linear algebra such as the QR factorization of Vandermonde matrices, cf. [5], 20.

These results show that in computational applications it is important to construct WAMs with low cardinalities and slowly increasing constants $C\left(A_{n}\right)$. We recall that is always possible to easily construct an AM on compact sets that admit a Markov polynomial inequality, as is shown in the key result, Theorem 5 of [8].

Here we are most interested in applications for which $K \subset \mathbb{R}^{d}$ is real. In this case there are known to be wide classes of such compacts, for example convex bodies, and more generally sets satisfying an interior cone condition, but the best known bounds for the cardinality of the resulting meshes grow like $\mathcal{O}\left(n^{r d}\right)$, where $r$ is the exponent of the Markov inequality (typically $r=2$ in the real case). This means that, for example, for a 3-dimensional cube or ball one should work with $\mathcal{O}\left(n^{6}\right)$ points, a number that becomes practically intractable already for relatively small values of $n$ (recall that the number of points determines the number of rows of the Least Squares (non-sparse) matrices). But already in dimension two, working with $\mathcal{O}\left(n^{4}\right)$ points makes the construction of polynomial approximants at moderate values of $n$ computationally rather expensive.

The properties of WAMs listed above (concerning finite unions and products) suggest an alternative: we can obtain good meshes, even on complicated geometries, if we are able to compute WAMs of low cardinality on standard compact "pieces". For example, it is immediate to get a $\mathcal{O}\left(n^{d}\right)$ WAM with $C\left(A_{n}\right)=\mathcal{O}\left(\log ^{d}(n)\right)$ for the $d$-dimensional cube, as the tensor-product of 1-dimensional Fekete (or Chebyshev) points. 
In this paper we introduce the notion of geometric WAM, that is, a WAM obtained by a geometric transformation of a suitable low-cardinality discretization mesh on some reference compact set, like the $d$-dimensional cube. Such geometric WAMs, as well as those obtained by finite unions and products, can be used directly for discrete Least Squares approximation, as well as for the extraction of good interpolation points by means of the Approximate Fekete Points algorithm described in [5], 20, on compact sets with various geometries.

In Section 2 we illustrate the idea of geometric WAMs by several examples in $\mathbb{R}^{2}$, the disk, triangles, trapezoids, and polygons. In Section 3 we prove a general result on the asymptotics of approximate Fekete points extracted from WAMs by the greedy algorithm in [5], 20]. Finally, in Section 4 we present some numerical results concerning discrete Least Squares approximation and interpolation at Approximate Fekete Points on geometric WAMs.

\section{Geometric WAMs}

Let $K$ and $Q$ be compact subsets of $\mathbb{R}^{d}$,

$$
\mathbf{t}: Q \rightarrow K \text { a surjective map }
$$

and $\left\{S_{n}\right\}$ a sequence of discrete subsets of $Q$. A geometric WAM of $K$ is a sequence

$$
A_{n}=\mathbf{t}\left(S_{n}\right)
$$

where the defining properties of a WAM, (1) - (3), stem from the geometric structure of $K, Q, \mathbf{t}$, and $S_{n}$.

To make this still somewhat vague notion more precise, we give some illustrative examples in $\mathbb{R}^{2}$, where the reference compact $Q$ is a rectangle.

2.1. The disk. A geometric WAM of the disk can be immediately obtained by working with polar coordinates, i.e., by considering the map

$$
\begin{aligned}
\mathbf{t}:[0,1] \times[0,2 \pi] & \rightarrow K=\left\{\mathbf{x}:\|\mathbf{x}\|_{2} \leq 1\right\} \\
\mathbf{t}(r, \phi) & =(r \cos \phi, r \sin \phi) .
\end{aligned}
$$

We now state and prove the following

Proposition 1. The sequence of polar grids

$$
S_{n}=\left\{\left(r_{j}, \phi_{k}\right)\right\}_{j, k}=\left\{\frac{1}{2}+\frac{1}{2} \cos \frac{j \pi}{n}, 0 \leq j \leq n\right\} \times\left\{\frac{2 \pi k}{2 n+1}, 0 \leq k \leq 2 n\right\}
$$

gives a WAM $A_{n}=\mathbf{t}\left(S_{n}\right)$ of the unit disk, such that $C\left(A_{n}\right)=\mathcal{O}\left(\log ^{2}(n)\right)$ and $\operatorname{card}\left(A_{n}\right)=2 n^{2}+n+1$.

Proof. Observe that given a polynomial $p \in \mathbb{P}_{n}^{2}$, when restricted to the disk in polar coordinates, $q(r, \phi)=p(\mathbf{t}(r, \phi))$, becomes a polynomial of degree $n$ in $r$ for any fixed $\phi$, and a trigonometric polynomial of degree $n$ in $\phi$ for any fixed $r$. Recall that $n+1$ Chebyshev-Lobatto points are near-optimal for 1-dimensional polynomial interpolation, and $2 n+1$ equally spaced points are near-optimal for trigonometric interpolation, both having a Lebesgue constant $\mathcal{O}(\log (n))$. Now, for every $p \in \mathbb{P}_{n}^{2}$ we can write

$$
\left|p\left(x_{1}, x_{2}\right)\right|=|q(r, \phi)|=|p(r \cos \phi, r \sin \phi)| \leq c_{1} \log (n) \max _{j}\left|q\left(r_{j}, \phi\right)\right|
$$



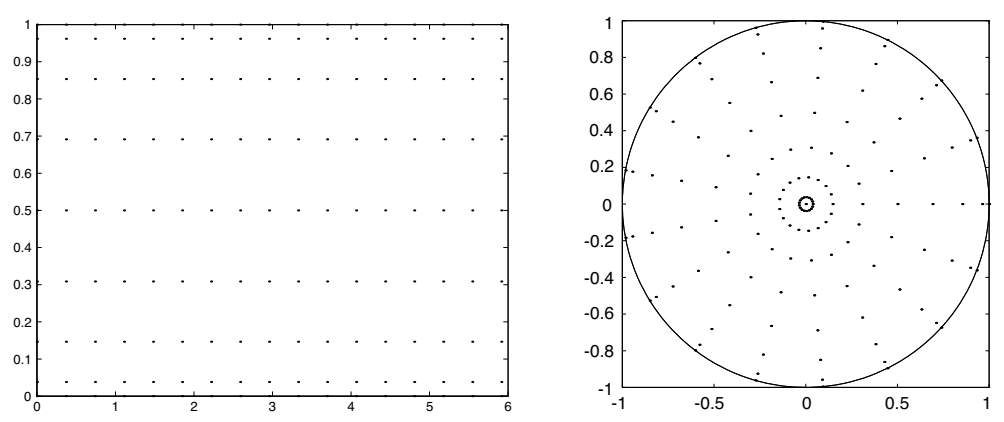

Figure 1. The polar grid and the corresponding geometric WAM of degree $n=8$ for the unit disk.

where $c_{1}$ is independent of $\phi$, since the $\left\{r_{j}\right\}$ are the $n+1$ Chebyshev-Lobatto points in $[0,1]$. Further,

$$
\left|q\left(r_{j}, \phi\right)\right| \leq c_{2} \log (n) \max _{k}\left|q\left(r_{j}, \phi_{k}\right)\right|
$$

where $c_{2}$ is independent of $j$, since the $\left\{\phi_{k}\right\}$ are $2 n+1$ equally spaced points in $[0,2 \pi]$. Thus

$$
\left|p\left(x_{1}, x_{2}\right)\right| \leq c_{1} c_{2} \log ^{2}(n) \max _{j, k}\left|q\left(r_{j}, \phi_{k}\right)\right|, \quad \forall\left(x_{1}, x_{2}\right) \in K,
$$

i.e., $A_{n}=\mathbf{t}\left(S_{n}\right)=\left\{\left(r_{j} \cos \phi_{k}, r_{j} \sin \phi_{k}\right)\right\}$ is a WAM for the disk with $C\left(A_{n}\right)=$ $\mathcal{O}\left(\log ^{2}(n)\right)$. We conclude by observing that the number of distinct points in $\mathbf{t}\left(S_{n}\right)$ is, due to the fact that $r_{n}=0, \operatorname{card}\left(S_{n}\right)-2 n=(n+1)(2 n+1)-2 n=2 n^{2}+n+1$.

In Figure 11 we display the polar grid $S_{n}$ and the WAM $A_{n}$ in the unit disk for $n=8$ (153 points and 137 points, respectively). In view of the structure of $S_{n}$ and $\mathbf{t}$, the points of the geometric WAM cluster at the boundary and at the center of the disk. Note that this technique can also be used to construct geometric WAMs for annuli and even a ball in higher dimensions. However, we will not pursue this here.

2.2. Polynomial maps. In the case that $\mathbf{t}$ is a polynomial map, we can give the following general proposition.

Proposition 2. Let the sequence of discrete subsets $\left\{B_{n}\right\}$ be a WAM of $Q$, and $\mathbf{t}: Q \rightarrow K$ a surjective polynomial map of degree $k$, i.e., $\mathbf{t}(\mathbf{y})=\left(t_{1}(\mathbf{y}), \ldots, t_{d}(\mathbf{y})\right)$ with $t_{j} \in \mathbb{P}_{k}^{d}$. Then $A_{n}=\mathbf{t}\left(S_{n}\right)$, with $S_{n}=B_{k n}$, is a WAM of $K$ such that $C\left(A_{n}\right)=C\left(B_{k n}\right)$.

Proof. Observing that for every $\mathbf{x} \in K$ there exists some $\mathbf{y} \in Q$ such that $\mathbf{x}=\mathbf{t}(\mathbf{y})$, and that for every $p \in \mathbb{P}_{n}^{d}$ the composition $p(\mathbf{t}(\cdot))$ is a polynomial of degree $\leq k n$, we have

$$
|p(\mathbf{x})|=|p(\mathbf{t}(\mathbf{y}))| \leq C\left(B_{k n}\right)\|p(\mathbf{t}(\cdot))\|_{B_{k n}}=C\left(B_{k n}\right)\|p\|_{\mathbf{t}\left(B_{k n}\right)} .
$$



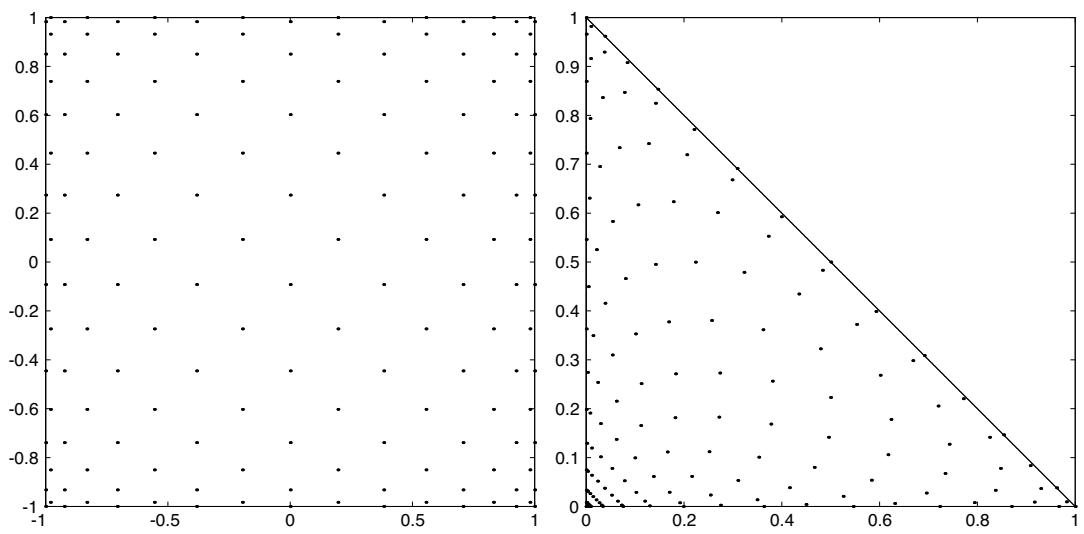

Figure 2. The Padua points of degree $2 n=16$ and the corresponding geometric WAM of degree $n=8$ for the unit simplex.

2.2.1. Triangles. We begin by observing that for the square $Q=[-1,1]^{2}$ several WAMs $B_{n}$ formed by good interpolation points are known, namely

- tensor-products of 1-dimensional near-optimal interpolation points, such as Chebyshev-Lobatto points, Gauss-Lobatto points, and others;

- the Padua points, recently studied in [4, 7], that are near-optimal for total-degree interpolation.

All of these WAMs have $C\left(B_{n}\right)=\mathcal{O}\left(\log ^{2}(n)\right)$, but the Padua points, have minimal cardinality $N=\operatorname{dim}\left(\mathbb{P}_{n}^{2}\right)=(n+1)(n+2) / 2$, whereas the cardinality of tensor-product points is $(n+1)^{2}=\operatorname{dim}\left(\mathbb{P}_{n}^{1} \otimes \mathbb{P}_{n}^{1}\right)$. We recall that, given the 1-dimensional Chebyshev-Lobatto points,

$$
\mathcal{C}_{n+1}=\{\cos (j \pi / n), 0 \leq j \leq n\},
$$

the Padua points of degree $n$ are given by the union of two Chebyshev-Lobatto grids:

$$
B_{n}=\operatorname{Pad}_{n}=\left(\mathcal{C}_{n+1}^{\text {odd }} \times \mathcal{C}_{n+2}^{\text {even }}\right) \cup\left(\mathcal{C}_{n+1}^{\text {even }} \times \mathcal{C}_{n+2}^{\text {odd }}\right) \subset \mathcal{C}_{n+1} \times \mathcal{C}_{n+2} .
$$

There is a simple quadratic map from the square to any triangle with vertices $\mathbf{u}=\left(u_{1}, u_{2}\right), \mathbf{v}=\left(v_{1}, v_{2}\right), \mathbf{w}=\left(w_{1}, w_{2}\right)$, namely the Duffy transformation (cf. [1])

$$
\mathbf{t}(\mathbf{y})=\frac{1}{4}(\mathbf{v}-\mathbf{u})\left(1+y_{1}\right)\left(1-y_{2}\right)+\frac{1}{2}(\mathbf{w}-\mathbf{u})\left(1+y_{2}\right)+\mathbf{u},
$$

which collapses one side of the square (here $y_{2}=1$ ) onto a vertex of the triangle (here w). By this map, the Padua points $B_{2 n}$ of the square (cf. (10) are transformed to the WAM $A_{n}=\mathbf{t}\left(B_{2 n}\right)$ of the triangle, with constants $C\left(A_{n}\right)=\mathcal{O}\left(\log ^{2}(2 n)\right)=$ $\mathcal{O}\left(\log ^{2}(n)\right)$. The number of distinct points in $\mathbf{t}\left(B_{2 n}\right)$ is $\operatorname{card}\left(A_{n}\right)=\operatorname{card}\left(B_{2 n}\right)-$ $\operatorname{card}\left(\mathcal{C}_{2 n+1}^{\text {odd }}\right)+1=\operatorname{dim}\left(\mathbb{P}_{2 n}^{2}\right)-(n+1)=2 n^{2}+2 n$. In Figure 2 we display the WAMs $B_{2 n}$ in the square and $A_{n}$ in the unit simplex for $n=8$ (153 points and 144 points, respectively). In view of the properties of the Padua points, the points of the geometric WAM cluster at the sides and especially at the vertices of the simplex. 

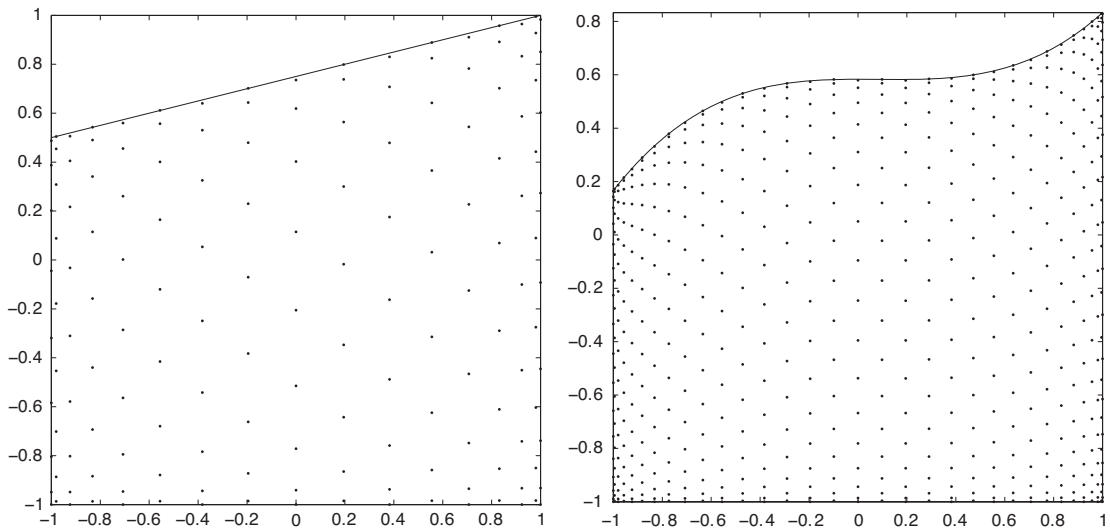

FiguRE 3. Examples of geometric WAMs of degree $n=8$ for a linear and a cubic trapezoid.

2.2.2. Polynomial trapezoids. We consider here bidimensional compact sets of the form

$$
K=\left\{\mathbf{x}=\left(x_{1}, x_{2}\right): a \leq x_{1} \leq b, g_{1}\left(x_{1}\right) \leq x_{2} \leq g_{2}\left(x_{1}\right)\right\},
$$

where $g_{1}, g_{2} \in \mathbb{P}_{\nu}^{1}$. A polynomial map $\mathbf{t}$ of degree $k=\nu+1$ from the square $Q=[-1,1]^{2}$ onto $K$ is

$$
t_{1}(\mathbf{y})=\frac{b-a}{2} y_{1}+\frac{b+a}{2}, t_{2}(\mathbf{y})=\frac{g_{2}\left(t_{1}\right)-g_{1}\left(t_{1}\right)}{2} y_{2}+\frac{g_{2}\left(t_{1}\right)+g_{1}\left(t_{1}\right)}{2} .
$$

Observe that a triangle could be treated (up to an affine transformation) as a degenerate linear trapezoid.

By Proposition 2, the Padua points $B_{k n}$ of the square are mapped to the WAM $A_{n}=\mathbf{t}\left(B_{k n}\right)$ of the polynomial trapezoid, with $C\left(A_{n}\right)=\mathcal{O}\left(\log ^{2}(k n)\right)$ and $\operatorname{card}\left(A_{n}\right) \leq \operatorname{card}\left(B_{k n}\right)=\operatorname{dim}\left(\mathbb{P}_{k n}^{2}\right)=(k n+1)(k n+2) / 2$.

In Figure 3 we show the WAMs $A_{n}=\mathbf{t}\left(B_{2 n}\right)$ and $A_{n}=\mathbf{t}\left(B_{4 n}\right)$ obtained by mapping the Padua points onto a linear trapezoid (quadratic map) and a cubic trapezoid (quartic map), again for $n=8$ (the numbers of points are $231=\operatorname{dim}\left(\mathbb{P}_{20}^{2}\right)$ and $561=\operatorname{dim}\left(\mathbb{P}_{32}^{2}\right)$, respectively). As expected, we observe clustering at the sides and at the vertices of the trapezoids. Notice that it could happen that $\operatorname{card}\left(A_{n}\right)<$ $\operatorname{card}\left(B_{k n}\right)$, namely when the graphs of $g_{1}$ and $g_{2}$ intersect at some $x_{1} \in t_{1}\left(\mathcal{C}_{k n+1}\right)$ (the $k n+1$ Chebyshev-Lobatto points of $[a, b]$ ).

2.2.3. Finite unions: polygons. A relevant example concerning finite unions is given by polygons, which are very important in applications such as 2-dimensional computational geometry. As is known, any simple (no interlaced sides) and simply connected polygon with $m$ vertices can be subdivided into $m-2$ triangles, and this can be done by fast algorithms; cf. e.g. 17. Once this rough triangulation is at hand, we can immediately obtain a geometric WAM $A_{n}$ for the polygon by taking the union of the geometric WAMs constructed by mapping the Padua points on the triangles, with $C\left(A_{n}\right)=\mathcal{O}\left(\log ^{2}(n)\right)$ and $\operatorname{card}\left(A_{n}\right) \leq(m-2)\left(2 n^{2}+2 n\right)$, in view of the basic properties of WAMs and the results of Section 2.2.1. The points of the union WAM will cluster especially at sides and vertices that the triangles have in common. 

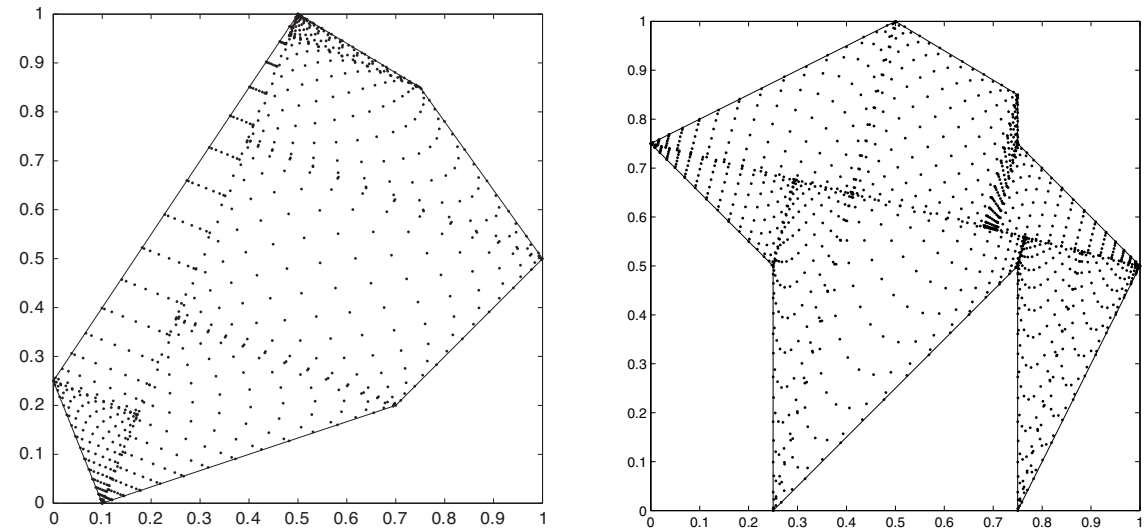

Figure 4. Examples of geometric WAMs of degree $n=8$ for a convex and a nonconvex polygon.

A similar approach is to subdivide into linear trapezoids, where we again obtain by finite union a geometric WAM $A_{n}$ for the polygon with $C\left(A_{n}\right)=\mathcal{O}\left(\log ^{2}(n)\right)$ and $\operatorname{card}\left(A_{n}\right)=\mathcal{O}\left(m n^{2}\right)$ (see Section 2.2.2). In Figure 4 we show geometric WAMs generated by the subdivision into linear trapezoids of a convex and a nonconvex polygon, for $n=8$. The method adopted, which works for a wide class of polygons, is that used for algebraic cubature in [19, where the trapezoidal panels are obtained simply by orthogonal projection of the sides on a fixed reference line (observe that the points cluster at the sides and at the line).

\section{Approximate Fekete points from WAMs}

In this section we return to the general setting of polynomial interpolation in several complex variables. Suppose that $K \subset \mathbb{C}^{d}$ is a compact polynomially determining set. If $\mathcal{B}_{n}=\left\{p_{1}, p_{2}, \ldots, p_{N}\right\}$ is a basis for $\mathbb{P}_{n}^{d}$, where $N:=\operatorname{dim}\left(\mathbb{P}_{n}^{d}\right)$ and $Z_{n} \subset K$ is a discrete subset of cardinality $N$, then

$$
\operatorname{vdm}\left(Z_{n} ; \mathcal{B}_{n}\right):=\operatorname{det}\left([p(\mathbf{a})]_{p \in \mathcal{B}_{n}, \mathbf{a} \in Z_{n}}\right)
$$

is called the corresponding Vandermonde determinant. If the determinant is nonzero then we may form the so-called fundamental Lagrange interpolating polynomials

$$
\ell_{\mathbf{a}}(\mathbf{z}):=\frac{\operatorname{vdm}\left(Z_{n} \backslash\{\mathbf{a}\} \cup\{\mathbf{z}\} ; \mathcal{B}_{n}\right)}{\operatorname{vdm}\left(Z_{n} ; \mathcal{B}_{n}\right)} .
$$

Indeed, then for any $f: K \rightarrow \mathbb{C}$ the polynomial (of degree $n$ )

$$
\Pi_{n}(\mathbf{z})=\sum_{\mathbf{a} \in Z_{n}} f(\mathbf{a}) \ell_{\mathbf{a}}(\mathbf{z})
$$

interpolates $f$ at the points of $Z_{n}$, i.e., $\Pi_{n}(\mathbf{a})=f(\mathbf{a}), \mathbf{a} \in Z_{n}$. A set of points $F_{n} \subset K$ which maximize $\operatorname{vdm}\left(Z_{n} ; \mathcal{B}_{n}\right)$ as a function of $Z_{n}$, are called Fekete points of degree $n$ for $K$ and have the special property that $\left\|\ell_{\mathbf{a}}\right\|_{K}=1$ (and hence Lebesgue 
constant bounded by $N$ ) and provide a very good (often excellent) set of interpolation points for $K$. However, they are typically very difficult to compute, even for moderate values of $n$.

For any $\mathbb{P}_{n}^{d}$ determining subset $A_{n} \subset K$ (thought of as a sufficiently good discrete model of $K$ ) the algorithm introduced by Sommariva and Vianello in 20] and studied by Bos and Levenberg in 5] selects, in a simple and efficient manner, a subset $\mathcal{F}_{n} \subset A_{n}$ of Approximate Fekete Points, and hence provides a practical alternative to true Fekete points, $F_{n}$. The optimization problem is nonlinear, and large-scale already for moderate values of $n$, but the algorithm is able to give an approximate solution using only standard tools of numerical linear algebra.

We sketch here the algorithm, in a Matlab-like notation. The goal is to extract a maximum volume square submatrix from the rectangular $N \times \operatorname{card}\left(A_{n}\right)$ Vandermonde matrix

$$
V=[p(\mathbf{a})]_{p \in \mathcal{B}_{n}, \mathbf{a} \in A_{n}},
$$

where the polynomial basis and the array of points have been (arbitrarily) ordered. The core is given by the following iteration:

Algorithm greedy (max volume submatrix of a matrix $V \in \mathbb{R}^{N \times M}, M>N$ )

- $\quad$ ind $=[]$;

- $\quad$ for $k=1, \ldots, N$

- "select the largest norm column $\operatorname{col}_{i_{k}}(V)$ "; ind $=\left[i n d, i_{k}\right]$;

- "subtract from every column of $V$ its orthogonal projection onto $\operatorname{col}_{i_{k}}$ end;

which works when $V$ is full rank and gives an approximate solution to the NP-hard maximum volume problem; cf. 9]. Then, we can extract the Approximate Fekete Points

$$
\mathcal{F}_{n}=A_{n}(\text { ind })=\left(A_{n}\left(i_{1}\right), \ldots, A_{n}\left(i_{N}\right)\right) .
$$

The algorithm can be conveniently implemented by the well-known QR factorization with column pivoting, originally proposed by Businger and Golub in 1965 [6], and used, for example, by the Matlab "mldivide" or "\" operator in the solution of underdetermined linear systems; cf. [16.

Some remarks on the polynomial basis $\mathcal{B}_{n}$ are in order. First note that if $\mathcal{B}_{n}^{\prime}:=$ $\left\{q_{1}, \ldots, q_{N}\right\}$ is some other basis of $\mathbb{P}_{n}^{d}$, then there is a transition matrix $T_{N} \in \mathbb{C}^{N \times N}$ so that $\mathcal{B}_{n}^{\prime}=\mathcal{B}_{n} T_{N}$. It is then easy to verify that

$$
\operatorname{vdm}\left(Z_{n} ; \mathcal{B}_{n}^{\prime}\right)=\operatorname{det}\left(T_{N}\right) \operatorname{vdm}\left(Z_{n} ; \mathcal{B}_{n}\right) \text {. }
$$

Hence, true Fekete points do not depend on the basis used and also the Lagrange polynomials $\ell_{\mathbf{a}}$ of (15) are independent of the basis. Moreover, if $A_{n}$ and $B_{n}$ are two point sets for which

$$
\left|\operatorname{vdm}\left(A_{n}, \mathcal{B}_{n}\right)\right| \geq C\left|\operatorname{vdm}\left(B_{n}, \mathcal{B}_{n}\right)\right|
$$

for some constant $C$, then the same inequality holds using the basis $\mathcal{B}_{n}^{\prime}$, i.e.,

$$
\left|\operatorname{vdm}\left(A_{n}, \mathcal{B}_{n}^{\prime}\right)\right| \geq C\left|\operatorname{vdm}\left(B_{n}, \mathcal{B}_{n}^{\prime}\right)\right|
$$

since both sides scale by the same factor.

The greedy algorithm described above is in general affected by the basis. But this not withstanding, in the theorem below we show that if the initial set $A_{n} \subset K$ 
is a WAM, then the so selected approximate Fekete points, using any basis, and the true Fekete points for $K$ both have the same asymptotic distribution.

We first introduce some notation. By

$$
\left|\operatorname{vdm}\left(\mathbf{z}_{1}^{(n)}, \ldots, \mathbf{z}_{N}^{(n)}\right)\right|
$$

we will mean the Vandermonde determinant computed using the standard monomial basis. Further, we set $m_{n}$ to be the sum of the degrees of the $N$ monomials of degree at most $n$, i.e., $m_{n}=d n N /(d+1)$.

Then it is known (see [25]) that if $F_{n}=\left\{\mathbf{f}_{1}^{(n)}, \mathbf{f}_{2}^{(n)}, \ldots, \mathbf{f}_{N}^{(n)}\right\} \subset K$ is a set of true Fekete points for $K$ the limit

$$
\lim _{n \rightarrow \infty}\left|\operatorname{vdm}\left(\mathbf{f}_{1}^{(n)}, \ldots, \mathbf{f}_{N}^{(n)}\right)\right|^{1 / m_{n}}=: \tau(K)
$$

exists and is called the transfinite diameter of $K$.

Theorem 1. Suppose that $K \subset \mathbb{C}^{d}$ is compact, non-pluripolar, polynomially convex and regular (in the sense of Pluripotential theory) and that for $n=1,2, \ldots, A_{n} \subset K$ is a WAM. Let $\left\{\mathbf{b}_{1}^{(n)}, \mathbf{b}_{2}^{(n)}, \ldots, \mathbf{b}_{N}^{(n)}\right\}$ be the Approximate Fekete Points selected from $A_{n}$ by the greedy algorithm of [20] described above, using any basis $\mathcal{B}_{n}$. Then

(1) $\lim _{n \rightarrow \infty}\left|\operatorname{vdm}\left(\mathbf{b}_{1}^{(n)}, \ldots, \mathbf{b}_{N}^{(n)}\right)\right|^{1 / m_{n}}=\tau(K)$, the transfinite diameter of $K$ and

(2) the discrete probability measures $\mu_{n}:=\frac{1}{N} \sum_{j=1}^{N} \delta_{\mathbf{b}_{j}^{(n)}}$ converge weak-* to the pluripotential-theoretic equilibrium measure $d \mu_{K}$ of $K$.

Remark. For $K=[-1,1], d \mu_{[-1,1]}=\frac{1}{\pi} \frac{1}{\sqrt{1-x^{2}}} d x$; for $K$ the unit circle $S^{1}, d \mu_{S^{1}}=$ $\frac{1}{2 \pi} d \theta$. If $K \subset \mathbb{R}^{d} \subset \mathbb{C}^{d}$ is compact, then $K$ is automatically polynomially convex. We refer the reader to [13] for other examples and more on complex pluripotential theory.

Note also that a set of true Fekete points $F_{n}$ is also a WAM and hence we may take $A_{n}=F_{n}$, in which case the algorithm will select $B_{n}=F_{n}$ (there is no other choice) and so the true Fekete points must necessarily also have these two properties.

Proof. We suppose that $F_{n}=\left\{\mathbf{f}_{1}^{(n)}, \mathbf{f}_{2}^{(n)}, \ldots, \mathbf{f}_{N}^{(n)}\right\} \subset K$ is a set of true Fekete points for $K$. Suppose further that $\left\{\mathbf{t}_{1}^{(n)}, \mathbf{t}_{2}^{(n)}, \ldots, \mathbf{t}_{N}^{(n)}\right\} \subset A_{n}$ is a set of true Fekete points of degree $n$ for $A_{n}$ and that $\ell_{i}^{(n)}$ are the corresponding Lagrange polynomials. Then,

$$
\left\|\ell_{i}^{(n)}\right\|_{K} \leq C\left(A_{n}\right)\left\|\ell_{i}^{(n)}\right\|_{A_{n}}=C\left(A_{n}\right) .
$$

It follows that the associated Lebesgue constants

$$
\Lambda_{n}:=\max _{\mathbf{z} \in K} \sum_{i=1}^{N}\left|\ell_{i}^{(n)}(\mathbf{z})\right| \leq N C\left(A_{n}\right)
$$

and hence, since $C\left(A_{n}\right)$ is of polynomial growth,

$$
\lim _{n \rightarrow \infty} \Lambda_{n}^{1 / n}=1 .
$$

By Theorem 4.1 of [2]

$$
\lim _{n \rightarrow \infty}\left|\operatorname{vdm}\left(\mathbf{t}_{1}^{(n)}, \ldots, \mathbf{t}_{N}^{(n)}\right)\right|^{1 / m_{n}}=\tau(K) .
$$


By Zaharjuta's famous result [25], we also have

$$
\lim _{n \rightarrow \infty}\left|\operatorname{vdm}\left(\mathbf{f}_{1}^{(n)}, \ldots, \mathbf{f}_{N}^{(n)}\right)\right|^{1 / m_{n}}=\tau(K) .
$$

Further, by 9 , we have (cf. the remarks on bases preceeding the statement of the Theorem)

$$
\left|\operatorname{vdm}\left(\mathbf{b}_{1}^{(n)}, \ldots, \mathbf{b}_{N}^{(n)}\right)\right| \geq \frac{1}{N !}\left|\operatorname{vdm}\left(\mathbf{t}_{1}^{(n)}, \ldots, \mathbf{t}_{N}^{(n)}\right)\right|
$$

and hence

$$
\begin{aligned}
\left|\operatorname{vdm}\left(\mathbf{f}_{1}^{(n)}, \mathbf{f}_{2}^{(n)}, \ldots, \mathbf{f}_{N}^{(n)}\right)\right| & \geq\left|\operatorname{vdm}\left(\mathbf{b}_{1}^{(n)}, \mathbf{b}_{2}^{(n)}, \ldots, \mathbf{b}_{N}^{(n)}\right)\right| \\
& \geq \frac{1}{N !}\left|\operatorname{vdm}\left(\mathbf{t}_{1}^{(n)}, \mathbf{t}_{2}^{(n)}, \ldots, \mathbf{t}_{N}^{(n)}\right)\right| .
\end{aligned}
$$

Thus by (18) and (19) we have

$$
\lim _{n \rightarrow \infty}\left|\operatorname{vdm}\left(\mathbf{b}_{1}^{(n)}, \mathbf{b}_{2}^{(n)}, \ldots, \mathbf{b}_{N}^{(n)}\right)\right|^{1 / m_{n}}=\tau(K)
$$

as

$$
\lim _{n \rightarrow \infty}(N !)^{1 / m_{n}}=1 .
$$

The final statement, that $\mu_{n}$ converges weak-* to $d \mu_{K}$ then follows by the main result of Berman and Boucksom [1] (see also [3]).

\section{Numerical Results}

In this section, we present a suite of numerical tests on certain compact sets $K \subset \mathbb{R}^{2}$ that we believe are useful in applications, concerning discrete least squares approximation on geometric WAMs and polynomial interpolation at Approximate Fekete Points extracted from them. The tests concern some of the 2-dimensional compact sets discussed in Section 2, i.e., the unit disk, the unit simplex, a cubic trapezoid and a nonconvex polygon; see Figures 1 14 . All the tests were done in Matlab (see [16]), by an Intel-Centrino Duo T-2400 processor with 1 Gb RAM.

In order to compute the Approximate Fekete Points, we actually used a refined version of the greedy algorithm of the previous section, which is sketched below.

\section{Algorithm greedy with iterative QR refinement of the basis}

- take the Vandermonde matrix $V$ in (17);

- $V_{0}=V^{t} ; T_{0}=I$

- $\quad$ for $k=0, \ldots, s-1$

- $\quad V_{k}=Q_{k} R_{k} ; U_{k}=\operatorname{inv}\left(R_{k}\right)$;

- $\quad V_{k+1}=V_{k} U_{k} ; T_{k+1}=T_{k} U_{k}$; end ;

- $b=(1, \ldots, 1)^{t} ; \quad$ (the choice of $b$ is irrelevant in practice)

- $w=V_{s}^{t} \backslash b ;$ ind $=\operatorname{find}(w \neq 0) ; \mathcal{F}_{n}=A_{n}($ ind $)$.

The greedy algorithm is implemented directly by the last row above (in Matlab), irrespectively of the actual value of the vector $b$, and produces a set of Approximate Fekete Points $\mathcal{F}_{n}$.

The for loop above implements a change of polynomial basis from $\left(p_{1}, \ldots, p_{N}\right)$ to basis $\left(q_{1}, \ldots, q_{N}\right)=\left(p_{1}, \ldots, p_{N}\right) T_{s}$ which is nearly orthogonal with respect to the discrete inner product $(f, g)=\sum_{\mathbf{a} \in A_{n}} f(\mathbf{a}) g(\mathbf{a})$, whose main aim is to cope with possible numerical rank-deficiency and severe ill-conditioning arising with 

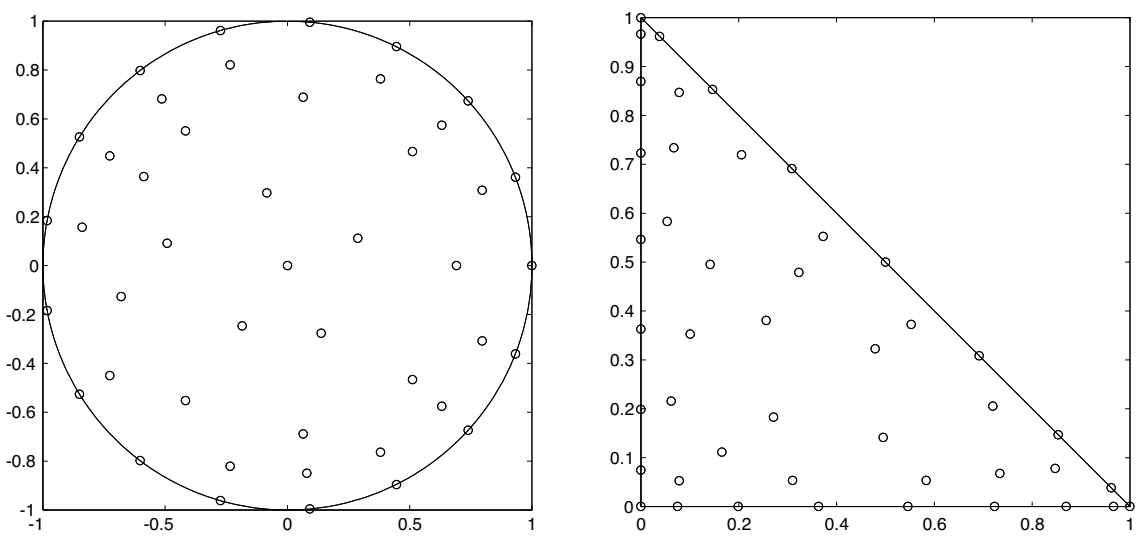

Figure 5. The 45 Approximate Fekete Points of degree $n=8$ extracted from the geometric WAMs for the disk and the simplex.

TABLE 1. Cardinalities of different point sets in the unit disk (AFP $=$ Approximate Fekete Points).

\begin{tabular}{|c|cccccc|}
\hline points & $n=5$ & $n=10$ & $n=15$ & $n=20$ & $n=25$ & $n=30$ \\
\hline AM & 2032 & 31700 & 159692 & 503868 & 1229072 & 2547452 \\
WAM & 60 & 220 & 480 & 840 & 1300 & 1860 \\
AFP & 21 & 66 & 136 & 231 & 351 & 496 \\
\hline
\end{tabular}

nonorthogonal bases (usually $s=1$ or $s=2$ iterations suffice); for a complete discussion of this algorithm we refer the reader to [20].

In Figures 5 17 we display the Approximate Fekete Points of degree $n=8$ extracted from the geometric WAMs of Figures 14.4. The computational advantage of working with a Weakly Admissible Mesh instead of an Admissible Mesh is shown in Table 1, where we show the cardinalities of the relevant discrete sets in the case of the disk. We recall that, following Theorem 5 of $[\underline{8}$, it is always possible to construct an AM for a real $d$-dimensional compact set that admits a Markov polynomial inequality with exponent $r$, by intersection with a uniform grid of stepsize $\mathcal{O}\left(n^{-r d}\right)$. In particular, convex compact sets have $r=2$, and it is easily seen from the Markov polynomial inequality $\|\nabla p(x)\|_{2} \leq n^{2}\|p\|_{\infty}$ valid for every $p \in \mathbb{P}_{n}^{2}$ and $x$ in the disk, and the proof of Theorem 5 of $[8$, that it is sufficient to take a stepsize $h<1 / n^{2}$. The cardinality of the corresponding AM is then $\mathcal{O}\left(n^{4}\right)$, to be compared with the $\mathcal{O}\left(n^{2}\right)$ cardinality of the geometric WAM (see Section 2.1). For example, at degree $n=30$ an AM for the disk has more than 2 millions points, whereas the geometric WAM has less than 2 thousands points.

In Table 2 we compare, for the two test functions below, the errors (in the uniform norm) of discrete least squares approximation on the AM and on the WAM of the disk, and of interpolation at the Approximate Fekete Points extracted from the WAM (with two refinement iterations). The test functions exhibit different regularity: the first is analytic entire and the second is $C^{1}$ but has a singularity in the second derivatives at the origin:

- test function 1: $f\left(x_{1}, x_{2}\right)=\cos \left(x_{1}+x_{2}\right)$,

- test function 2: $f\left(x_{1}, x_{2}\right)=\left(x_{1}^{2}+x_{2}^{2}\right)^{3 / 2}$. 

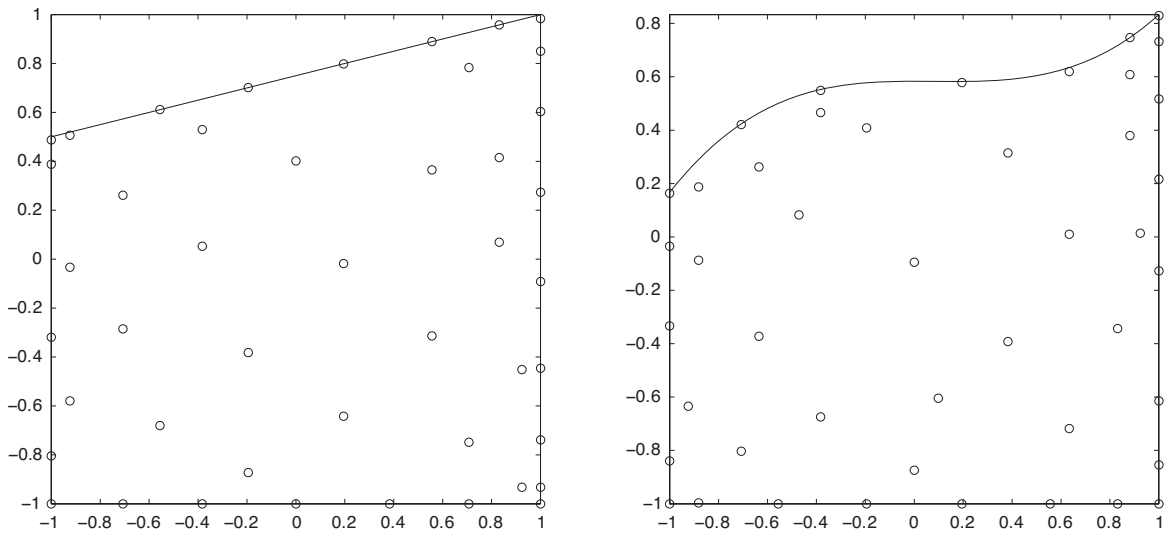

Figure 6. The 45 Approximate Fekete Points of degree $n=8$ extracted from the geometric WAMs for a linear and a cubic trapezoid.

TABLE 2. Uniform errors of polynomial approximations on different point sets in the unit disk, for the two test functions above; * means computational failure due to large dimension (see Table 1).

\begin{tabular}{|lc|cccccc|}
\hline & points & $n=5$ & $n=10$ & $n=15$ & $n=20$ & $n=25$ & $n=30$ \\
\hline test 1 & LS AM & $9 \mathrm{E}-4$ & $3 \mathrm{E}-10$ & $*$ & $*$ & $*$ & $*$ \\
& LS WAM & $7 \mathrm{E}-4$ & $2 \mathrm{E}-10$ & $4 \mathrm{E}-15$ & $4 \mathrm{E}-15$ & $8 \mathrm{E}-15$ & $9 \mathrm{E}-15$ \\
& interp AFP & $7 \mathrm{E}-4$ & $2 \mathrm{E}-10$ & $2 \mathrm{E}-15$ & $1 \mathrm{E}-15$ & $1 \mathrm{E}-15$ & $1 \mathrm{E}-15$ \\
\hline test 2 & LS AM & $2 \mathrm{E}-2$ & $2 \mathrm{E}-3$ & $*$ & $*$ & $*$ & $*$ \\
& LS WAM & $1 \mathrm{E}-2$ & $8 \mathrm{E}-4$ & $3 \mathrm{E}-4$ & $1 \mathrm{E}-4$ & $6 \mathrm{E}-5$ & $3 \mathrm{E}-5$ \\
& interp AFP & $2 \mathrm{E}-2$ & $1 \mathrm{E}-3$ & $1 \mathrm{E}-3$ & $4 \mathrm{E}-4$ & $2 \mathrm{E}-4$ & $1 \mathrm{E}-4$ \\
\hline
\end{tabular}

The Vandermonde matrices have been constructed using the Logan-Shepp orthogonal basis; cf. 14. Both the least squares and the interpolation polynomial coefficients have been computed by the standard Matlab "backslash" solver, and the errors have been evaluated on a suitable control mesh.

Notice that with the AM we have computational failure ("out of memory") in our computing system already at degree $n=15$, due to the large cardinality of the discrete set; see Table1 The least squares error on the WAM is close to that on the AM (when comparable), which shows that geometric WAMs are a good choice for polynomial approximation, with a low computational cost. It is worth observing that in the theoretical estimate (4) we even have $C\left(A_{n}\right) \sqrt{\operatorname{card}\left(A_{n}\right)}=\mathcal{O}\left(n^{2}\right)$ for the AM, and $C\left(A_{n}\right) \sqrt{\operatorname{card}\left(A_{n}\right)}=\mathcal{O}\left(n \log ^{2}(n)\right)$ for the WAM, but we recall that these are overestimates, the term $\sqrt{\operatorname{card}\left(A_{n}\right)}$ being in some way "artificial" (cf. Theorem 2 of $[8]$ ).

The following tables are devoted to numerical tests on the other domains. First, in Table 3 we show the Lebesgue constants of the Approximate Fekete Points extracted from the geometric WAMs described in Section 2. Again, the growth is much slower than that of the theoretical bound (5). 

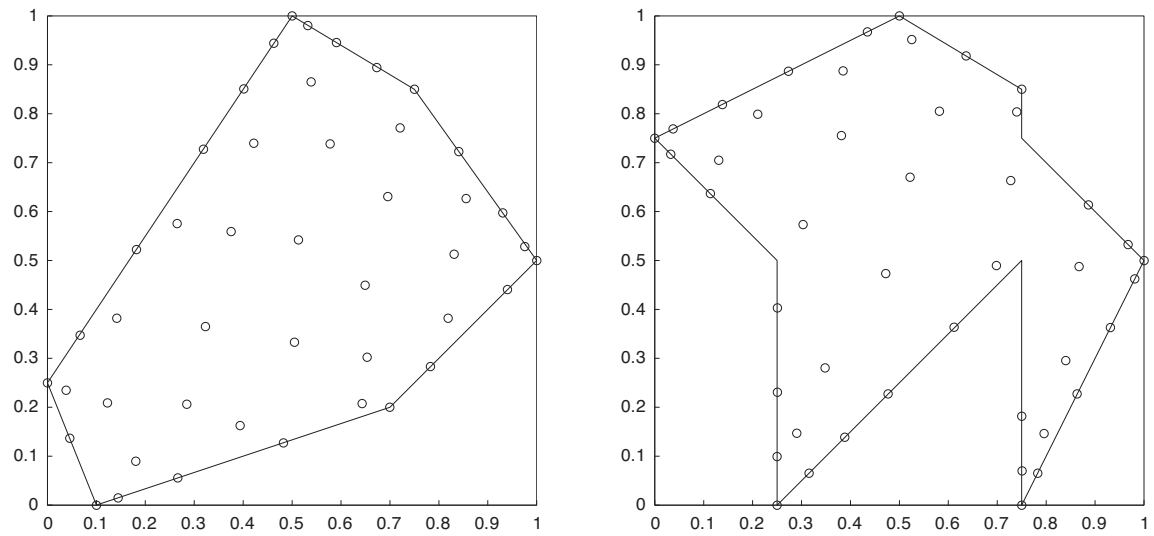

Figure 7. The 45 Approximate Fekete Points of degree $n=8$ extracted from the geometric WAMs fora convex and a nonconvex polygon.

As for the simplex, the Lebesgue constant of our Approximate Fekete Points points is larger than that of the best points known in the literature. The case of the simplex has been widely studied and several specialized approaches have been proposed for the computation of Fekete or other good interpolation points, due to the relevance in the numerical treatment of PDEs by spectral-element and high order finite-element methods; see e.g. [12, [18, 22, [24] and references therein.

On the other hand, our method for computing Approximate Fekete Points via geometric WAMs is quite general and flexible, since it allows us to work on a wide class of compact sets and, differently from other computational approaches, up to reasonably high interpolation degrees. The good quality of the discrete sets used for least squares approximation and for interpolation is evidenced by Tables 46 . We observe that the singularity of the second test function is in the interior of the domains, apart from the simplex where it is located at a vertex (where the discrete points cluster). This explains the better results with the simplex for this function. In the case of the polygon, a change of variables is made in order to put the problem in the reference square $[-1,1]^{2}$. The availability of good interpolation points in compact sets with various geometries has a number of potential applications. One, for example, is connected to numerical cubature. Indeed, when the moments of the underlying polynomial basis are known [19, cubature weights associated to the Approximate Fekete Points can be computed as a by-product of the algorithm, simply by using the moments vector as right-hand side $b$. This gives an algebraic cubature formula, that can be used directly, or as a starting point towards the computation of a minimal formula, by the method developed in 23. Another relevant application concerns the numerical treatment of PDEs, where there is renewed interest in global polynomial methods, such as collocation and discrete least squares, over general domains (see, e.g., 15]). Recently, Approximate Fekete Points have been successfully used for discrete least squares discretization of elliptic equations 26. Moreover, Approximate Fekete Points for polygons could play a role in connection with discretization methods on polygonal (nonsimplicial) meshes (see, e.g., 21] and references therein). 
TABLE 3. Numerically evaluated Lebesgue constants (nearest integer) of the Approximate Fekete Points extracted from the geometric WAMs on different compact sets. Bases used: Logan-Shepp [14] for the disk and Dubiner [10] for the simplex (both orthogonal with respect to the Lebesgue measure), product Chebyshev otherwise (all with 2 refinement iterations).

\begin{tabular}{|c|cccccc|}
\hline set & $n=5$ & $n=10$ & $n=15$ & $n=20$ & $n=25$ & $n=30$ \\
\hline disk & 6 & 16 & 31 & 48 & 102 & 127 \\
simplex & 5 & 16 & 26 & 57 & 62 & 86 \\
linear trap & 6 & 19 & 34 & 37 & 31 & 54 \\
cubic trap & 6 & 16 & 35 & 46 & 40 & 78 \\
conv polyg & 5 & 12 & 35 & 34 & 55 & 75 \\
nonconv polyg & 6 & 9 & 26 & 42 & 54 & 75 \\
\hline
\end{tabular}

TABLE 4. Uniform errors of polynomial approximations in the unit simplex, for the two test functions above.

\begin{tabular}{|lc|cccccc|}
\hline & points & $n=5$ & $n=10$ & $n=15$ & $n=20$ & $n=25$ & $n=30$ \\
\hline test 1 & LS WAM & $7 \mathrm{E}-7$ & $7 \mathrm{E}-15$ & $2 \mathrm{E}-15$ & $4 \mathrm{E}-15$ & $5 \mathrm{E}-15$ & $5 \mathrm{E}-15$ \\
& interp AFP & $2 \mathrm{E}-6$ & $2 \mathrm{E}-14$ & $1 \mathrm{E}-15$ & $2 \mathrm{E}-15$ & $6 \mathrm{E}-15$ & $3 \mathrm{E}-15$ \\
\hline test 2 & LS WAM & $7 \mathrm{E}-4$ & $5 \mathrm{E}-6$ & $4 \mathrm{E}-7$ & $8 \mathrm{E}-8$ & $2 \mathrm{E}-8$ & $7 \mathrm{E}-9$ \\
& interp AFP & $8 \mathrm{E}-4$ & $2 \mathrm{E}-5$ & $2 \mathrm{E}-6$ & $6 \mathrm{E}-7$ & $7 \mathrm{E}-8$ & $2 \mathrm{E}-8$ \\
\hline
\end{tabular}

TABle 5. As in Table 4, for the cubic trapezoid of Figure 3.

\begin{tabular}{|lc|cccccc|}
\hline & points & $n=5$ & $n=10$ & $n=15$ & $n=20$ & $n=25$ & $n=30$ \\
\hline test 1 & LS WAM & $2 \mathrm{E}-3$ & $6 \mathrm{E}-9$ & $6 \mathrm{E}-14$ & $2 \mathrm{E}-15$ & $4 \mathrm{E}-15$ & $5 \mathrm{E}-15$ \\
& interp AFP & $6 \mathrm{E}-3$ & $1 \mathrm{E}-8$ & $1 \mathrm{E}-13$ & $3 \mathrm{E}-15$ & $3 \mathrm{E}-15$ & $5 \mathrm{E}-15$ \\
\hline test 2 & LS WAM & $3 \mathrm{E}-2$ & $3 \mathrm{E}-3$ & $9 \mathrm{E}-4$ & $5 \mathrm{E}-4$ & $2 \mathrm{E}-4$ & $2 \mathrm{E}-4$ \\
& interp AFP & $6 \mathrm{E}-2$ & $5 \mathrm{E}-3$ & $9 \mathrm{E}-4$ & $7 \mathrm{E}-4$ & $2 \mathrm{E}-4$ & $2 \mathrm{E}-4$ \\
\hline
\end{tabular}

TABle 6 . As in Table 5 for the nonconvex polygon of Figure 4 and the two test functions $f\left(2 x_{1}-1,2 x_{2}-1\right)$ above.

\begin{tabular}{|cc|cccccc|}
\hline & points & $n=5$ & $n=10$ & $n=15$ & $n=20$ & $n=25$ & $n=30$ \\
\hline test 1 & LS WAM & $5 \mathrm{E}-4$ & $3 \mathrm{E}-10$ & $5 \mathrm{E}-15$ & $3 \mathrm{E}-14$ & $4 \mathrm{E}-14$ & $3 \mathrm{E}-13$ \\
& interp AFP & $5 \mathrm{E}-4$ & $5 \mathrm{E}-10$ & $2 \mathrm{E}-15$ & $3 \mathrm{E}-15$ & $4 \mathrm{E}-15$ & $5 \mathrm{E}-15$ \\
\hline test 2 & LS WAM & $2 \mathrm{E}-2$ & $3 \mathrm{E}-3$ & $8 \mathrm{E}-4$ & $3 \mathrm{E}-4$ & $1 \mathrm{E}-4$ & $9 \mathrm{E}-5$ \\
& interp AFP & $4 \mathrm{E}-2$ & $3 \mathrm{E}-3$ & $7 \mathrm{E}-4$ & $3 \mathrm{E}-4$ & $2 \mathrm{E}-4$ & $1 \mathrm{E}-4$ \\
\hline
\end{tabular}




\section{REFERENCES}

[1] R. Berman and S. Boucksom, D. Witt Nyström, Fekete points and convergence towards equilibrium measures on complex manifolds, arXiv:0907.2820 (Acta Math., to appear).

[2] T. Bloom, L. Bos, C. Christensen and N. Levenberg, Polynomial interpolation of holomorphic functions in $\mathbb{C}$ and $\mathbb{C}^{n}$, Rocky Mtn. J. Math. 22 (1992), 441-470. MR.1180711 (93i:32016)

[3] T. Bloom, L. Bos, N. Levenberg and S. Waldron, On the convergence of optimal measures, Constr. Approx. 32 (2010), no. 1, 159-179. MR2659752

[4] L. Bos, M. Caliari, S. De Marchi, M. Vianello and Y. Xu, Bivariate Lagrange interpolation at the Padua points: the generating curve approach, J. Approx. Theory 143 (2006), 15-25. MR.2271722(2007h:41001)

[5] L. Bos and N. Levenberg, On the approximate calculation of Fekete points: the univariate case, Electron. Trans. Numer. Anal. 30 (2008), 377-397. MR2487768 (2010a:41001)

[6] P.A. Businger and G.H. Golub, Linear least-squares solutions by Householder transformations, Numer. Math. 7 (1965), 269-276. MR0176590 (31:862)

[7] M. Caliari, S. De Marchi and M. Vianello, Algorithm 886: Padua2D: Lagrange Interpolation at Padua Points on Bivariate Domains, ACM Trans. Math. Software 35-3 (2008).

[8] J.P. Calvi and N. Levenberg, Uniform approximation by discrete least squares polynomials, J. Approx. Theory 152 (2008), 82-100. MR2419299 (2009c:32020)

[9] A. Civril and M. Magdon-Ismail, On selecting a maximum volume sub-matrix of a matrix and related problems, Theoretical Comp. Sci. 410 (2009), 4801-4811. MR2583677

[10] M. Dubiner, Spectral methods on triangles and other domains, J. Sci. Computing 6, no. 4 (1991), 345-390. MR:1154903 (92k:76061)

[11] M.G. Duffy, Quadrature over a pyramid or cube of integrands with a singularity at a vertex, SIAM J. Numer. Anal. 19 (1982), 1260-1262. MR679664 (83k:65020)

[12] J.S. Hesthaven and T. Warburton, Nodal discontinuous Galerkin methods algorithms, analysis, and applications, Texts in Applied Mathematics, 54, Springer, New York, 2008. MR2372235 (2008k:65002)

[13] M. Klimek, Pluripotential Theory, Oxford U. Press, 1991. MR.1150978 (93h:32021)

[14] B.F. Logan and L.A. Shepp, Optimal reconstruction of a function from its projections, Duke Math. J. 42 (1975), 645-659. MR0397240 (53:1099)

[15] Y. Maday, N.C. Nguyen, A.T. Patera and G.S.H. Pau, A general multipurpose interpolation procedure: the magic points, Commun. Pure Appl. Anal. 8 (2009), 383-404. MR2449115 (2009i:65016)

[16] The Mathworks, MATLAB documentation set, 2008 version, (http://www.mathworks.com).

[17] A. Narkhede and D. Manocha, Graphics Gems 5, Academic Press, 1995, pp. 394-397.

[18] R. Pasquetti and F. Rapetti, Spectral element methods on unstructured meshes: comparisons and recent advances, J. Sci. Comput. 27 (2006), 377-387. MR2285788 (2008a:65235)

[19] A. Sommariva and M. Vianello, Product Gauss cubature over polygons based on Green's integration formula, BIT Numerical Mathematics 47 (2007), 441-453. MR2334049(2008g:65046)

[20] A. Sommariva and M. Vianello, Computing approximate Fekete points by QR factorizations of Vandermonde matrices, Comput. Math. Appl. 57 (2009), 1324-1336. MR2512231

[21] N. Sukumar and E.A. Malsch, Recent advances in the construction of polygonal finite element interpolants, Arch. Comput. Methods Engrg. 13 (2006), 129-163. MR2283620|(2007m:65113)

[22] M.A. Taylor, B.A. Wingate and R.E. Vincent, An algorithm for computing Fekete points in the triangle, SIAM J. Numer. Anal. 38 (2000), 1707-1720. MR.1813252 (2001k:65042)

[23] M.A. Taylor, B.A. Wingate and L.P. Bos, A cardinal function algorithm for computing multivariate quadrature points, SIAM J. Numer. Anal. 45 (2007), 193-205. MR2285850 (2008i:65046)

[24] T. Warburton, An explicit construction of interpolation nodes on the simplex, J. Engrg. Math. 56 (2006), 247-262. MR2292675 (2008c:65022)

[25] V.P. Zaharjuta, Transfinite diameter, Chebyshev constants and capacity for compacts in $\mathbb{C}^{n}$, Math. USSR Sbornik 25 (1975), 350-364.

[26] P. Žitňan,, A stable collocation solution of the Poisson problems on planar domains, 2nd Dolomites Workshop on Constructive Approximation and Applications (DWCAA09), Alba di Canazei (Trento, Italy), Sept. 4-9 2009 (abstract online at: http://drna.di.univr.it). 
Department of Mathematics and Statistics, University of Calgary, Calgary, AlBerta, Canada T2N 1N4

Institut de Mathématiques de Toulouse, Université Paul Sabatier, 32062, Toulouse Cedex 9, France

Department of Mathematics, Indiana University, Bloomington, Indiana, 47405

Department of Pure and Applied Mathematics, University of Padova, 35121 Padova, ITALY

Department of Pure and Applied Mathematics, University of Padova, 35121 Padova, ITALY 